\title{
SMALL HANKEL OPERATORS ON DIRICHLET-TYPE SPACES AND APPLICATIONS
}

\author{
ZENGJIAN LOU AND RUISHEN QIAN
}

Abstract. In this paper, we characterize the boundedness and compactness of small Hankel operators on Dirichlet-type spaces $D_{\rho}$.

Mathematics subject classification (2010): 47B35, 30H99.

Keywords and phrases: Carleson measure, small Hankel operator, Dirichlet-type space.

\section{REFERENCES}

[1] A. Aleman, Hilbert spaces of analytic functions between the Hardy space and the Dirichlet space, Proc. Amer. Math. Soc. 115 (1992), 97-104.

[2] N. Arcozzi, R. Rochberg AND E. SAWyer, Carleson measures for analytic Besov spaces, Rev. Mat. Iberoamericana 18 (2002), 443-510.

[3] G. BaO, Z. Lou, R. Qian and H. Wulan, On multipliers of Dirichlet type spaces, Complex Anal. Oper. Theory. doi: 10.1007/s11785-015-0444-0, in press.

[4] D. Blasi And J. PaU, A charaterization of Besov type spaces and applications on Hankel type operators, Michigan Math. J. 56 (2008), 401-417.

[5] C. C. Cowen And B. D. MacCluer, Composition Operators on Spaces of Analytic Functions, CRC Press, Boca Raton, FL, 1995.

[6] P. Duren, Theory of $H^{p}$ Spaces, Academic Press, New York, 1970.

[7] J. Garnett, Bounded Analytic Functions, Academic Press, New York, 1981.

[8] P. Hu AND W. Zhang, Small Hankel operators on the Dirichlet-type spaces on the Unit ball of $C^{n}$, Acta Math. Sin. Engl. Ser. 20 (2004), 261-272.

[9] S. JANSON, Generalizations of Lipschitz spaces and an application to Hardy spaces and bounded mean oscillation, Duke Math. J. 47 (1980), 959-982.

[10] R. Kerman AND E. SAWYeR, Carleson measures and multipliers of Dirichlet-type spaces, Trans. Amer. Math. Soc. 309 (1988), 87-98.

[11] S. Li AND S. STEvić, Composition followed by differentiation between $H^{\infty}$ and $\alpha$-Bloch spaces, Houston J. Math. 35 (2009), 327-340.

[12] X. Liu, G. Chacón And Z. Lou, Characterizations of Dirichlet-type spaces, Complex Anal. Oper. Theory 9 (2015), 1269-1286.

[13] D. LuECKING, Representation and duality in weighted spaces of analytic functions, Indiana Univ. J. Math. 34 (1985), 319-336.

[14] R. QIAN AND Y. SHI, Inner function in Dirichlet type spaces, J. Math. Anal. Appl. 421 (2015), 18441854.

[15] R. Rochberg And S. Semmes, A decomposition theorem for BMO and applications, J. Funct. Anal. 67 (1986), 228-263.

[16] R. RochBerg AND Z. Wu, A new characterization of Dirichlet type spaces and applications, Illinois J. Math. 37 (1993), 101-122.

[17] S. STEVIĆ, On an integral-type operator from logarithmic Bloch-type and mixed-norm spaces to Bloch-type spaces, Nonlinear Anal. Theory, Methods and Applications, 71 (2009), 6323-6342.

[18] S. STEvić, Weighted differentiation composition operators from $H^{\infty}$ and Bloch spaces to $n$th weighted-type spaces on the unit disk, Appl. Math. Comput. 216 (2010), 3634-3641. 
[19] H. Wulan And J. Zhou, Decomposition theorems for $\mathscr{Q}_{K}$ spaces and applications, Forum Math. 26 (2014), 467-495.

[20] K. ZHU, Operator Theory in Function Spaces, American Mathematical Society, Providence, RI, 2007. 